\title{
Engineered disulfide bonds support the functional rotation mechanism of multidrug efflux pump AcrB
}

\author{
Markus A Seeger ${ }^{1,2,4}$, Christoph von Ballmoos ${ }^{2}$, Thomas Eicher ${ }^{1}$, Lorenz Brandstätter ${ }^{1}$, François Verrey ${ }^{1}$, \\ Kay Diederichs ${ }^{3} \&$ Klaas M Pos ${ }^{1}$
}

The AcrA-AcrB-TolC complex is the major multidrug efflux pump in Escherichia coli. The asymmetric structure of the trimeric inner-membrane component AcrB implies functional rotation of the monomers and a peristaltic mode of drug efflux. This mechanism suggests the occurrence of conformational changes in the periplasmic pore domain through the movements of subdomains during cycling of the monomers through the different states loose (L), tight $(\mathrm{T})$ and open $(\mathrm{O})$. We introduced cysteines at the interfaces of potentially moving subdomains, leading to disulfide bond formation as quantified by alkylation of free cysteines and MALDI-TOF analysis. Inhibition of pump function as a result of cross-linking caused increased susceptibility to noxious compounds and reduction of $\mathrm{N}$-phenylnaphthylamine efflux. Regain of function for impaired mutants was obtained upon exposure to the reducing agent DTT. The results support the presence of the asymmetric AcrB trimer in $E$. coli membranes and the functional rotation mechanism.

In Gram-negative bacteria, three-component multidrug efflux systems have an important role in the transport of noxious substances such as dyes, detergents, bile salts and diverse antibiotics out of the cell ${ }^{1}$. In Escherichia coli, the major multidrug efflux system is composed of the inner-membrane resistance nodulation (cell) division (RND) transporter AcrB, the outer-membrane channel TolC and the membrane fusion protein $\mathrm{AcrA}^{2-4}$. Crystal structures of all three individual components have been solved ${ }^{5-10}$.

Homotrimeric TolC is composed of an outer membrane-integrated 40- $\AA \beta$-barrel domain, with an inner diameter of $20 \AA$ and an $\alpha$-helical domain protruding $100 \AA$ into the periplasm. Structural and biochemical studies indicate that the proximal end of TolC has the ability to selectively open and close the channel ${ }^{5,11}$. AcrA interacts with both $\mathrm{AcrB}$ and $\mathrm{TolC}$, and is therefore proposed to act as a linker protein of the tripartite efflux system ${ }^{12-14}$. Homotrimeric AcrB operates as a proton and drug antiporter, and it is responsible for the drug specificity and energy transduction of the tripartite efflux system ${ }^{15,16}$. Its three-dimensional structure has been solved in a three-fold symmetric conformation ${ }^{6,7,17,18}$ and, most recently, in an asymmetric conformation, which is thought to represent the physiologically relevant form ${ }^{8,9}$.

An AcrB monomer contains 12 transmembrane $\alpha$-helices (TM1 to TM12, see Supplementary Fig. 1 online) and 2 large periplasmic loops consisting of the TolC docking domain (DN and DC subdomains), which is located most distant from the membrane plane, and the pore domain, composed of subdomains PN1, PN2, PC1 and PC2.
The AcrB monomers in the asymmetric trimer structure were suggested to represent consecutive steps of a transport cycle and were designated loose $(\mathrm{L})$, tight $(\mathrm{T})$ and open $(\mathrm{O})^{9}$. The proposed cycling of each monomer through the different conformational states $\mathrm{L}, \mathrm{T}$ and $\mathrm{O}$ and back to $\mathrm{L}$ results in the formation of an alternate access tunnel within the pore domain. In the $\mathrm{L}$ monomer, the tunnel has a lateral access about $15 \AA$ above the membrane plane and leads to the central part of the pore domain. In the T monomer, the tunnel is extended and includes a hydrophobic pocket that has been shown to bind minocycline and doxorubicin ${ }^{8,9}$. Moreover, recent highresolution structure information on the asymmetric AcrB trimer indicates the presence of a second tunnel in this monomer, leading from the TM8-TM9 groove at the membrane surface toward the hydrophobic substrate binding pocket ${ }^{19}$. In the $\mathrm{O}$ monomer, the lateral pathways are closed, but another tunnel leading from the now closed binding pocket to the funnel and toward $\mathrm{TolC}$ is present. The assumed cycling mechanism creates an alternate access pathway for drug transport, where the gradual opening and closing of the tunnel access and drug binding pocket resembles the function of a peristaltic pump? The conformational cycling and functional rotation, with strong analogy to the mechanism of ATP synthesis by the $F_{1} F_{0}$ ATPase ${ }^{20}$, implies a concerted conformational change in each monomer that is dependent on the conformational state of the neighboring monomers. In this report, we use site-directed cysteine cross-linking to provide evidence for the occurrence of asymmetric AcrB trimer in the E. coli membrane and for the requirement of conformational changes in the

\footnotetext{
${ }^{1}$ Institute of Physiology and Zurich Centre for Integrative Human Physiology (ZIHP), University of Zurich, Winterthurerstrasse 190, CH-8057 Zürich, Switzerland. ${ }^{2}$ Institute of Microbiology, Swiss Federal Institute of Technology (ETH), Wolfgang-Pauli-Strasse 10, CH-8093 Zürich, Switzerland. ${ }^{3}$ Department of Biology, University of Konstanz, Universitätsstrasse 10, M647, D-78457 Konstanz, Germany. ${ }^{4}$ Current address: Department of Pharmacology, University of Cambridge, Tennis Court Road, Cambridge CB2 1PD, United Kingdom. Correspondence should be addressed to K.M.P. (kmpos@access.uzh.ch).
} 
AcrB pore domain to accomplish drug efflux. The results support the recently proposed functional rotation hypothesis of AcrB during drug transport 8,9 .

\section{RESULTS}

\section{Rationale and construction of cysteine-substituted AcrB}

The proposed functional rotation of the AcrB trimer ${ }^{8,9}$ suggests a concerted cycling of the monomers through the different states $\mathrm{L}$, $\mathrm{T}$ and $\mathrm{O}$, energized by the proton motive force. The alternate states include different conformations of the transmembrane domain and of the subdomains PN1, PN2, PC1 and PC2, which constitute the pore domain of the AcrB monomers (Supplementary Fig. 1). As a consequence, the distance variation of amino acid residues in the moving subdomains is expected to be considerable (Table 1 and Supplementary Fig. 2 online). This structural prerequisite was used to test the conformational cycling of the monomers by the introduction of cysteine pairs at the interface of potentially moving and rigid subdomains (or loops or helices) in a cysteine-free AcrB background (AcrB_cl; Table 1). Both AcrA and TolC are naturally devoid of cysteines, so the introduced cysteines are unique in the functional tripartite pump unit. During the transport cycle, introduced cysteines that approach sufficiently to facilitate disulfide cross-linking in the oxidative environment of the periplasm will cause cessation of subdomain movements, cycling and, hence, antibiotic efflux.

We constructed ten mutant versions of AcrB_cl, each of which harbored one cysteine pair. Five of these pairs were introduced at positions that would allow the establishment of intermolecular crosslinks between either the intermonomer connecting loop ('loop') and the $\mathrm{PC} 1$ subdomain (mutants Q229C T583C and Q229C_R586C) or between the loop and the DC subdomain (S233C_Q726C, I235C_K728C and V225C_A777C) (Fig. 1). Four other introduced cysteine pairs were expected to form intramolecular cross-links at the PN1-PN2 subdomain interface (S132C and A294C), between the PC2 subdomain and the C-terminal part of transmembrane helix 7 (TM7; S562C and T837C) or between the PN2 subdomain and TM1 (V32C_N298C and V32C_A299C) (Fig. 1). With this set of mutants, we expected to obtain specific cross-links in the L conformer (PN1PN2 and loop-PC1 cross-link), the T conformer (PN2-TM1) and the O conformer (PC2-TM7). As a control, we used a mutant containing substitutions R558C (PC2 subdomain) and E839C (TM7). According to the asymmetric structure, the introduced thiol groups of the last mutant are not closer than $10 \AA$ from each other in $\mathrm{L}, \mathrm{T}$ or $\mathrm{O}$, and therefore no cross-linking is expected. In contrast, the double-cysteine mutants S233C_Q726C, I235C_K728C and V225C_A777C acted as positive functional controls. Cross-linking in any conformation was expected (Table 1), but transport function and the ability to confer drug resistance to $E$. coli were assumed to be unaffected, because the introduced cysteines are in the putative nonmoving subdomain DC and loop. We analyzed the cysteine mutants with respect to qualitative physical cross-linking via SDS-PAGE and western blot analysis, and quantitatively by MALDI-TOF analysis. With respect to function, mutants were analyzed for the ability to confer drug resistance to the E. coli cell, and inhibition or regeneration of activity was further investigated with $\mathrm{N}$-phenylnaphthylamine (NPN) fluorescence spectroscopy.

\section{Cysteine mutant synthesis and detection of cross-links}

Cysteine-substituted AcrB_cl was synthesized in E. coli BW25113 $\triangle$ acrB, and cells harboring pET24a (expression vector) or pET24acrB_cl encoding AcrB_cl were used as a negative and positive control, respectively. All AcrB_cl derivatives were produced to comparable amounts in this low-expression system (see Methods), as indicated by
Table 1 Distances of residues and degree of disulfide cross-linking

\begin{tabular}{|c|c|c|c|c|}
\hline \multirow{3}{*}{$\begin{array}{l}\text { Linked subdomains }^{\mathrm{a}} \\
\text { cysteine residues }\end{array}$} & \multicolumn{3}{|c|}{ Distance between $\mathrm{S}_{\gamma}[\AA]^{\mathrm{b}}$} & \multirow{3}{*}{$\begin{array}{c}\text { Disulfide cros } \\
\text { links [\% }]^{c}\end{array}$} \\
\hline & \multicolumn{3}{|c|}{ Monomer } & \\
\hline & L & $T$ & 0 & \\
\hline \multicolumn{5}{|l|}{ PC2-TM7 (control) ${ }^{\mathrm{d}}$} \\
\hline R558C_E839C & 16.2 & 14.0 & 10.3 & $-9.5 \pm 4.7^{\mathrm{e}}$ \\
\hline \multicolumn{5}{|l|}{ PC2-TM7 } \\
\hline S562C_T837C & 10.9 & 10.7 & 3.3 & $15.4 \pm 1.3^{\mathrm{e}}$ \\
\hline \multicolumn{5}{|l|}{$P N 1-P N 2$} \\
\hline S132C_A294C & 6.3 & 17.5 & 11.1 & $41.6 \pm 0.6$ \\
\hline \multicolumn{5}{|l|}{ PN2-TM1 } \\
\hline V32C_N298C & 7.2 & 3.5 & 7.0 & $41.3 \pm 1.2$ \\
\hline V32C_A299C & 9.5 & 4.8 & 11.9 & $18.1 \pm 1.0$ \\
\hline \multicolumn{5}{|c|}{ Loop $-D C$ (functional control) } \\
\hline S233C_Q726C & 5.2 & 5.9 & 6.3 & $17.0 \pm 0.4$ \\
\hline 1235C_K728C & 5.0 & 5.0 & 5.1 & $23.8 \pm 0.6$ \\
\hline V225C_A777C & 5.1 & 5.2 & 5.3 & $80.2 \pm 1.3$ \\
\hline \multicolumn{5}{|l|}{ Loop-PCl } \\
\hline Q229C_T583C & 5.8 & 7.4 & 6.4 & $69.4 \pm 0.5$ \\
\hline Q229C_R586C & 5.5 & 7.8 & 6.7 & $46.4 \pm 0.5$ \\
\hline
\end{tabular}

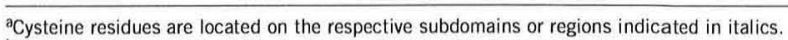
Cysteine residues are located on the respective subdomains or regions indicated in italics.
bwhen alanine was replaced by a cysteine, the introduced cysteine was allowed to adopt the 'When alanine was replaced by a cysteine, the introduced cysteine was allowed to adopt the
most likely rotamer conformation. cRelative amount of cysteines involved in disulfide crosslinking as determined by quantitative MALDI-TOF analysis. ${ }^{2}$ R558C_E839C cysteine substitutions were chosen as control. These cysteine residues are not expected to participate in cross-linking because of the long-range distance between these residues in all three monomers. The presence of cross-links in the PC2-TM7 control mutant could not be determined by MALDI-TOF analysis because the peak of the $\mathrm{N}$-methylmaleimide (NMM)-modified peptide overlapped with a peak from a peptide originating from $\mathrm{CNBr}$ digest of $\mathrm{AcrB}$ _cl. The disulfide cross-link content for the R558C_E839C and the S562C_T837C mutants was determined alternatively using radioactively labeled $\left[{ }^{14} \mathrm{C}\right] \mathrm{N}$-ethylmaleimide (NEM) (see Supplementary Methods). The results indicated $-9.5 \pm 4.7 \%$ and $16.5 \pm 1.4 \%$ cross-links, respectively, compared to the cysteine cross-link content of AcrB_cl. IIntermonomer connecting loop.

the presence of a major $\alpha$-AcrB immunoreactive band with an electrophoretic mobility corresponding to a protein of $100-110 \mathrm{kDa}$ (Fig. 2a). When SDS-PAGE and western blot analysis were carried out in the absence of reducing substances, the intermolecular cross-linking of the loop with the DC subdomain (S233C_Q726C, I235C_K728C or V225C_A777C) or with the PC1 subdomain (Q229C_T538C or Q229C_R586C) became apparent (Fig. 2b). The observed high molecular weight cross-link products were not present either in the single-cysteine mutants or when intramolecular cross-links where expected (AcrB_cl double mutants S562C_T837C, S132C_A294C, V32C_N298C and V32C_A299C). Particularly strong cross-linking was observed for the Q229C_T583C (loop-PC1) and V225C_A777C (loop-DC) mutants.

After overproduction of the AcrB_cl double mutants in E. coli C43(DE3) and affinity chromatography under oxic conditions, intermolecular cross-linking yielded high molecular weight species ( $>200 \mathrm{kDa}$; Fig. 2c, left) representing AcrB dimers and trimers. A faint band that probably corresponds to an AcrB dimer is also detected for our control mutant R558C_E839C; this is likely to result from the exposure of R558C (and E839C) at the protein surface, which could facilitate intermolecular cross-linking in the E. coli cell. Under reducing conditions, all mutants showed an electrophoretic mobility comparable to wild-type $\mathrm{AcrB}^{17}$ (Fig. 2c, right).

Intra- and intermolecular cross-link quantification

Intramolecular cross-linking was determined using quantitative MALDI-TOF analysis (Supplementary Fig. 3 online). The analysis 


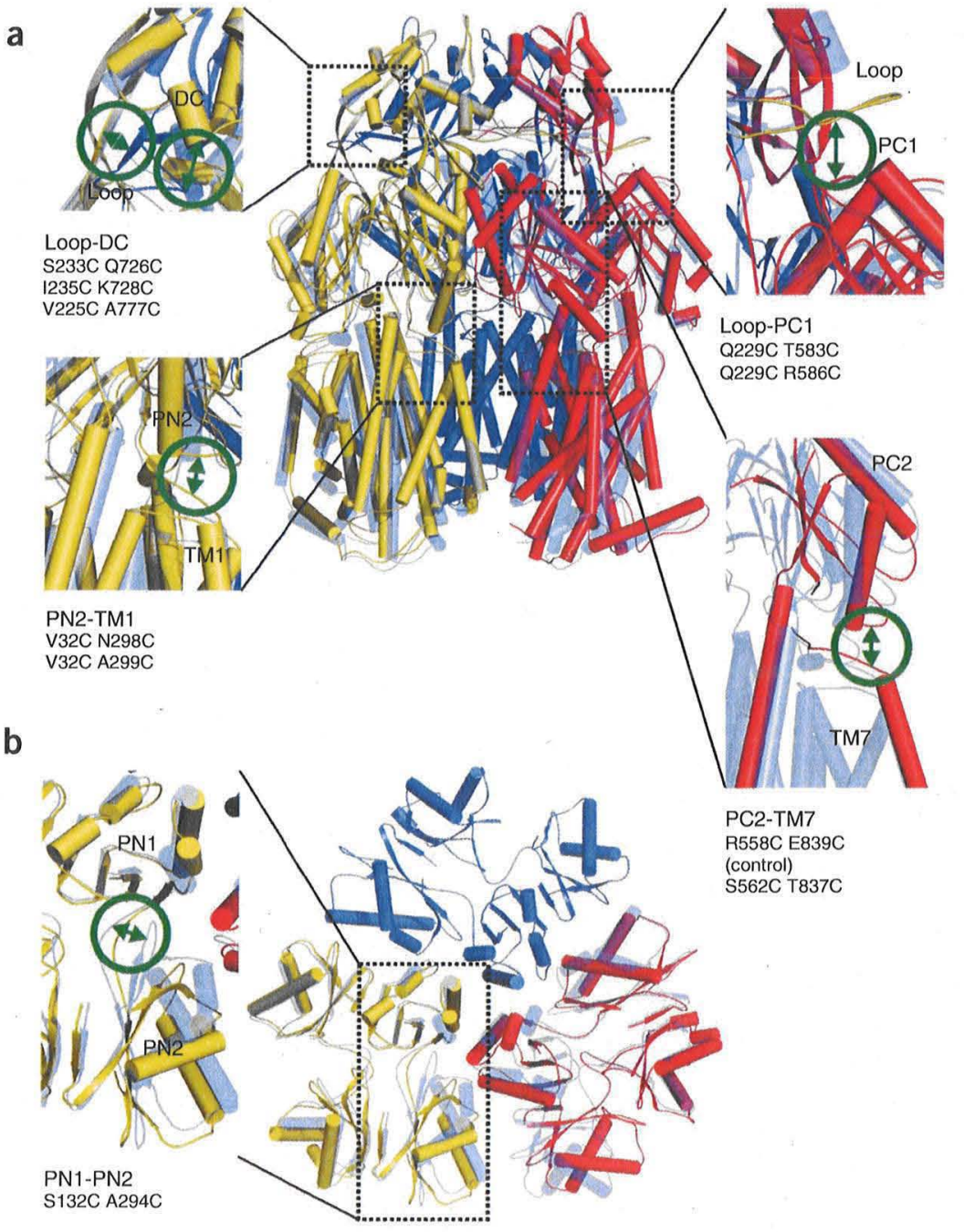

Figure 1 Visualization of the engineered disulfide bridges. The three AcrB monomers shown as cylinder presentations in blue $(L)$, yellow $(T)$ and red $(\mathrm{O})$ are superimposed onto the structure of the $L$ monomer, which is depicted in transparent blue. a depicts a side view of the entire AcrB trimer, and $\mathbf{b}$ represents a top view onto the pore domain. Enlarged views of the boxed regions (dashed lines) are shown at the left and right borders of the figure. The locations of the disulfide bridges are indicated by encircled arrows (green). Below the close-up views, the subdomains harboring the cysteine residues and the respective substitutions are indicated. Figure 1 and Supplementary Figure 4 were created using PyMOL (http://www.pymol.org).

$\beta$-sheet interaction (Fig. 1a). As expected, the negative control mutant R558C_E839C (PC2TM7 control) was devoid of disulfide crosslinks (Table 1).

\section{Subdomain cross-linking and drug susceptibility}

Cysteine mutants of AcrB_cl were analyzed with respect to their ability to confer drug resistance to $E$. coli BW25113 $\triangle$ acrB (Table 2 and Supplementary Fig. 2b). Cells containing the plasmid encoding AcrB_cl were slightly more susceptible (with a minimal inhibitory concentration (MIC) of about two-fold lower) compared to cells harboring AcrB wild-type protein (not shown).

Single-cysteine mutants all conferred approximately the same level of resistance to the E. coli cell as the cysteine-free AcrB control, with the exception of the single-cysteine mutant T583C, which for all substrates showed a consistent two-fold reduction in

clearly demonstrates the occurrence of intramolecular cysteine bridges in the double-cysteine mutants, with S562C_T837C linking the PC2 subdomain to TM7, S132C_A294C linking the PN1 and PN2 subdomains, and V32C_N298C and V32C_A299C linking.TM1 to the PN2 subdomain (Table 1). The PC2-TM7 cross-link occurs in $15.4 \pm$ $1.3 \%$ of all monomers, whereas in a substantially higher number of S132C_A294C monomers cross-linking was observed between the PN1-PN2 subdomains $(41.6 \pm 0.6 \%)$. Moreover, there was ample cross-linking between the PN2 subdomain and TM1 in the V32C_N298C mutant $(41.3 \pm 1.2 \%)$, and cross-links were also observed, albeit to a lesser extent, in the V32C_A299C mutant $(18.1 \pm 1.0 \%)$. The quantitative analysis furthermore confirmed the strong intermolecular cross-link formation between the loop and the PC1 subdomain in the double-cysteine AcrB_cl mutant Q229C_T583C of $69.4 \pm 0.6 \%$ and to a lesser extent in the Q229C_R586C (loop-PC1) mutant $(46.4 \pm 0.5 \%$; Table 1$)$. In the double-cysteine mutant V225C_A777C located on the putative nonmoving DC subdomain and the loop, extensive disulfide formation was observed (80.2 \pm $1.3 \%)$. Unexpectedly, the cysteine mutants S233C_Q726C and I235C_K728C showed relatively moderate cross-linking (17.0 $\pm 0.4 \%$ and $23.8 \pm 0.6 \%$, respectively), despite the expected proximity of the $S \gamma$ according to the structure $(5.0-6.3 \AA)$. On the other hand, this result might reflect the relative side chain inflexibility imposed by the parallel

MIC, and the N298C mutant, which showed a four- to eight-fold reduction in MIC. For this reason, we constructed a second loop-PC1 double mutant (both Gln229 and Arg586 to cysteine) and a second PN2-TM1 mutant (both Val32 and Ala299 to cysteine).

Escherichia coli cells synthesizing double-cysteine AcrB mutants causing cross-linking between the PC2 subdomain and TM7 (S562C_T837C), the PN1 and PN2 subdomains (S132C_A294C) or the loop and the PC1 subdomain (Q229C_T583C and Q229C_R586C) (Fig. 2b,c and Table 1) showed a distinctive increase in susceptibility of up to eight-fold for all drugs tested. The strongest effect was observed with the loop-PC1 (Q229C_T583C) and the PN1PN2 (S132C_A294C) cross-linked double-cysteine mutants, with $69.4 \%$ and $41.6 \%$ of the monomers cross-linked, respectively (Table 1). Strong MIC reduction was also observed with cells harboring the PC2-TM7 cross-link mutant despite the observed cross-linking of only $15.4 \%$ (Table 1 ). In contrast, only moderate MIC reduction was observed for the PN2-TM1 mutants. V32C_N298C (41.3\% crosslinking) was susceptible to TPP and berberine, whereas V32C_A299C (18.1\%) showed reduced resistance only to oxacillin.

Mutant R558C_E839C, which contained cysteines located on the PC2 subdomain and TM7, was used as a control, because the distances between these cysteines in all three AcrB monomer conformations (>10 ̊) was expected to prohibit cross-linking. Indeed, this mutant 

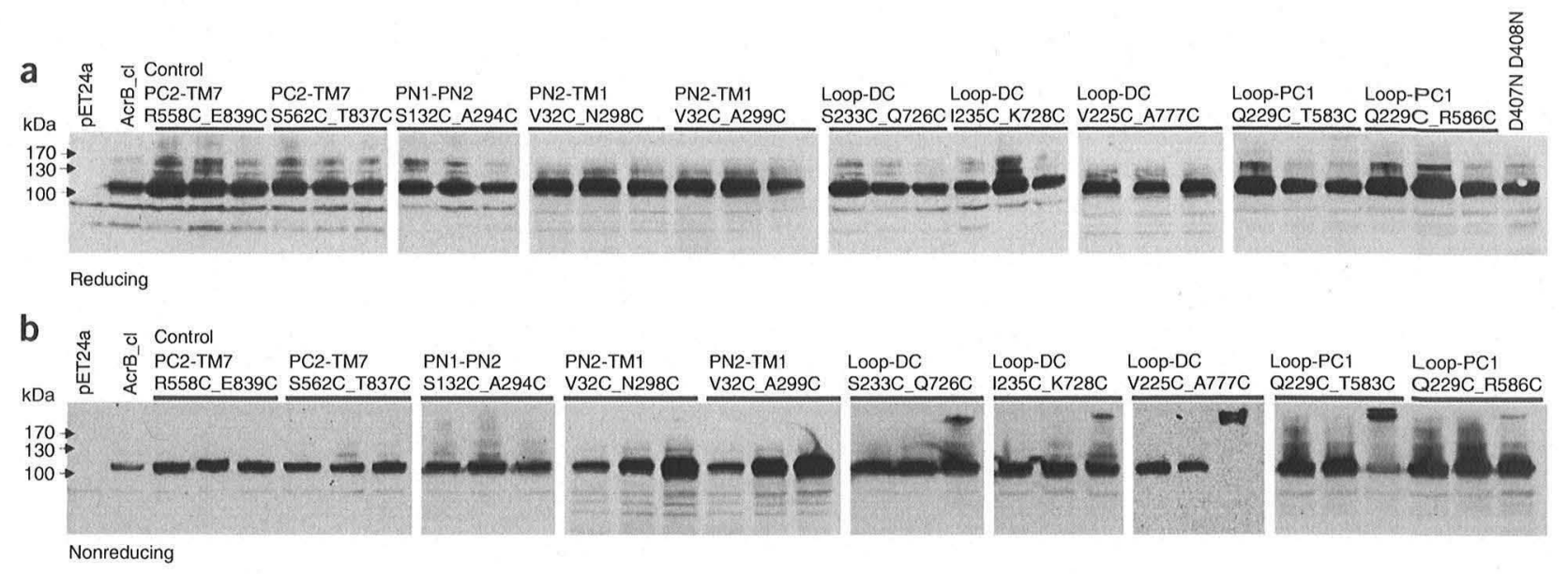

C
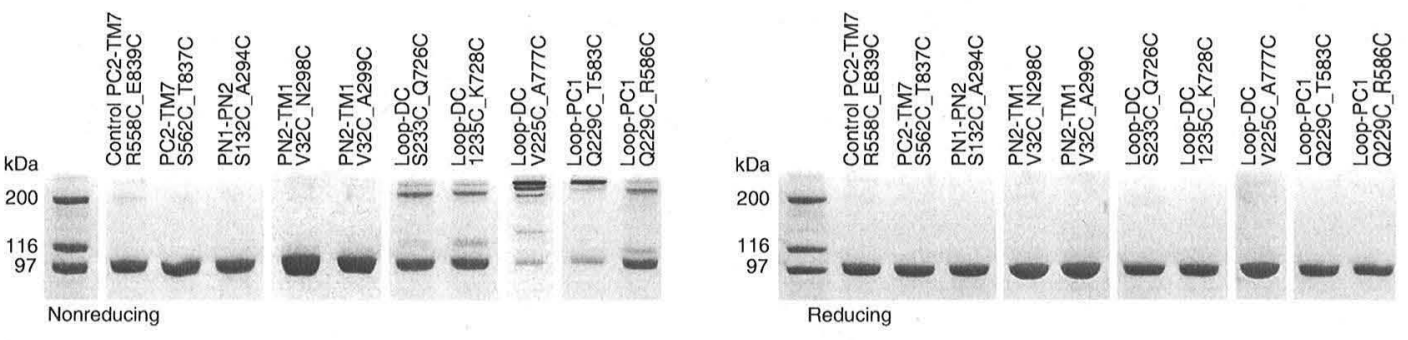

Figure 2 SDS-PAGE and western blot analysis of single- and double-cysteine mutants of AcrB_cl. Cysteines were introduced at the positions indicated. $(a, b)$ Western blot analysis under reducing (a) and nonreducing (b) conditions. Cysteine-substituted AcrB_cl proteins from E. coli BW25113 $\Delta a c r B$ harboring mutated pET24acrB_cl were probed with AcrB antiserum. The same batch of cells was used for activity measurements via $N$-phenylnaphthylamine (NPN) fluorescence spectroscopy and minimum inhibitory concentration (MIC) determination. As a control, E. coli BW25113 $\triangle a c r B$ was complemented with the expression vector (pET24a), with pET24acrB_cl encoding cysteine-free AcrB or with pET24acrB_D407N_D408N. For each cysteine-substituted AcrB_cl clone (beneath the black bar), the three lanes indicate from left to right the single-cysteine substituted AcrB_cl clones (starting with the most $\mathrm{N}$-terminal substituted residue first) and the double-cysteine substituted mutant AcrB_cl in the third lane. (c) Purified, mutant AcrB_cl clones were analyzed for the degree of cross-linking by SDS-PAGE under nonreducing or reducing conditions. Disulfide cross-linking between the loop and DC subdomain (S233C_Q726C, I235C_K728C and V225C_A777C) as well as between the loop and PC1 subdomain (Q229C_R586C and Q229C_T583C) was observed. All cross-linked mutants could be reduced to monomers (right). Molecular size markers are indicated on the left.

conferred the same drug resistance to the E. coli cell as AcrB_cl. For the V255C_A777C mutant, in which the cysteines were located on a putative rigid body comprising the loop and the DC subdomain, strong cross-linking $(80.2 \%)$ had no appreciable effect on the ability to confer resistance.

The inhibition of the efflux activity of the double-cysteine mutants leading to reduced MIC values is likely to be explained by the conformational restraints inflicted by the cross-links, which impede a conformational change. If this is true, reduction of the restraining disulfide bonds must lead to reactivation of the drug-pumping activity.

Disulfide cross-link reduction restores pump activity

Activity-constrained AcrB_cl double-cysteine mutants that showed reduced resistance levels and the V255C_A777C mutant, which showed no change in MIC, were further analyzed via NPN fluorescence spectroscopy (Fig. 3). NPN is strongly fluorescent in the hydrophobic environment of the bacterial inner membrane and is a cognate substrate of tripartite RND efflux systems ${ }^{21,22}$. Cells that produce AcrB_cl and the inactive double mutant D407N_D408N were used as positive (maximum quench) and negative (no quench) controls, respectively (Fig. 3a). Those AcrB double-cysteine mutants showing decreased drug-transport activities in the MIC determinations (including the V32C_N298C mutant) (Table 2) also showed reduced NPN efflux compared to the positive control with respect to the maximal efflux rate and the final steady-state level (Fig. $3 \mathbf{b}-\mathbf{e}$, yellow traces, and Supplementary Fig. 2c). Notably, the relative quantity of cross-link in the tested mutants (Table 1 and indicated in Fig. 3) seemed to correlate with the amount of inhibition observed. The inhibitory effect was most pronounced in the Q229C_T583C mutant, in which the loop was cross-linked with the PC1 subdomain (Fig. 3e). A distinct reduction of NPN efflux was also observed for the S132C_A294C mutant, with approximately 42\% cross-linking between the PN1 and PN2 subdomains (Fig. 3c), for the S562C_T837C mutant, with $15 \%$ cross-linking between the PC2 subdomain and TM7 (Fig. 3b), and for the V32C_N298C mutant, with $41 \%$ cross-linking between the PN2 subdomain and TM1.

Notably, activity was restored upon exposure to the reducing agent DTT (Fig. 3b-e, red traces) for all cross-linked mutants except V225C_A777C (Fig. 3f). This result indicates that the double-cysteine mutant proteins are inhibited mainly because of the disulfide crosslinks and not as a result of improper folding during synthesis of the protein. The transport stimulation of the S132C_A294 mutant upon addition of DTT (Fig. 3c) was distinct but rather modest, probably resulting from the impaired accessibility of DTT to the buried disulfide bridge within the pore domain. The recovery of transport activity of the V32C_N298C mutant was also expected to be limited, 
because the N298C substitution alone already affects the ability to transport drugs (Table 2). DTT did not stimulate the NPN efflux of AcrB_cl (Fig. 3a), the inactive mutant D407N_D408N (Fig. 3a) and the control mutant V225C_A777C (in which the loop was cross-linked with the DC subdomain)(Fig. 3f). The last result confirmed that the amount of cross-linking (80.2\%) had no effect on NPN efflux activity.

\section{DISCUSSION}

We recently solved an asymmetric structure of trimeric AcrB that suggests a functional rotation of the trimer in which the monomers run through the consecutive states $\mathrm{L}, \mathrm{T}$ and $\mathrm{O}$ of a drug-transport cycle, leading to an alternate access mechanism and a peristaltic mode of drug transport ${ }^{9}$.

In this study, we selected pairs of residues that, on the basis of the conformational cycling hypothesis, show great variation in distance depending on the conformational state, and substituted these residues with cysteines in a cysteine-free background. Formation of disulfide bonds requires a minimum distance between the thiol groups $(6.4 \AA)$ to guarantee the exclusion of water molecules between those atoms $s^{23,24}$. As described in Table 1 and Supplementary Figure 2, the distance between the $\mathrm{S} \gamma$ atoms of the introduced cysteines is dependent on the conformation of the monomer and varies between $16.2 \AA$ and $3.3 \AA$. The observed disulfide formation of the AcrB cysteine mutants (Table 1) is expected to represent the cross-link fraction within the native $E$. coli membrane, because the addition of $\mathrm{N}$-ethylmaleimide (NEM) to E. coli membranes prevented further disulfide formation during downstream manipulation steps.

The cysteines introduced in the PN1-PN2 subdomains, the PC2 subdomain and TM7, and the loop-PC1 subdomain approach sufficiently to cause cross-linking (Fig. 2 and Table 1 ) and as a consequence inhibit AcrA-AcrB-TolC activity (Fig. 3, Table 2 and Supplementary Fig. 2).

Moreover, NPN fluorescence spectroscopy data show a clear correlation between the reduced drug-transport activity and the degree of cross-linking (Fig. 3). Cross-link formation seems to exert conformational restraints on the subdomains involved and results in the inability to complete the cycle through the conformational states $\mathrm{L}$, $\mathrm{T}$ and $\mathrm{O}$ and back to $\mathrm{L}$, which is necessary for drug transport. Crosslink formation results in the observed inactivation of the pump; this was inferred because the presence of the reducing agent DTT largely restored drug efflux activity (Fig. 3 ). This regain of activity was found only in those cases in which independent subdomain movement was expected to be crucial according to the functional rotation mechanism. In cases in which subdomain movement is already constrained without cross-linking, as in the loop-DC mutant (V225C_A777C), the addition of DTT reduces disulfide bonds (Fig. 2) but does not stimulate NPN efflux (Fig. 3f).

Disulfide formation between the PC2 subdomain and TM7 in the S562C_T837C mutant strongly supports the existence of the $\mathrm{O}$ monomer and, consequently, the existence of the asymmetric AcrB structure in vivo. In the $\mathrm{O}$ conformation, the distance between the $S \gamma$ atoms is $3.3 \AA$, whereas in the other conformations the distances are $>10 \AA$. For the PC2-TM7 mutant, reduction of the MIC values (Table 2) and to a lesser extent the inhibition of NPN efflux activity (Fig. 3b) seem to be more severe than expected for this degree of cross-linking (Table 1 and Supplementary Fig. 2). One possible, but by no means exclusive, model would be that the $\mathrm{O}$ conformation is trapped within the trimer, whereas the other monomers can adapt either the $\mathrm{L}$ or the $\mathrm{T}$ conformation but cannot, owing to structural restraints, be in the $\mathrm{O}$ conformation as well. Hence, the amount of cross-linking observed in the PC2-TM7 mutant (15\%)
Table 2 Drug resistance of $E$. coli BW25113 $\Delta$ acrB expressing acrB mutant genes

\begin{tabular}{|c|c|c|c|c|c|}
\hline \multirow[b]{2}{*}{$\begin{array}{l}\text { Plasmid or } \\
\text { mutation }\end{array}$} & \multicolumn{5}{|c|}{ MIC $\left[\mu \mathrm{g} \mathrm{ml}^{-1}\right]$} \\
\hline & $\begin{array}{l}\text { Tetraphenyl- } \\
\text { phosphonium }\end{array}$ & $\begin{array}{c}\text { Rhodamine } \\
\text { 6G }\end{array}$ & Berberine & $\begin{array}{l}\text { Erythro- } \\
\text { mycin }\end{array}$ & Oxacillir \\
\hline \multicolumn{6}{|l|}{ Plasmid $^{a}$} \\
\hline pET24a & 3.125 & 2 & 64 & 4 & 2 \\
\hline pET24acrB_cl & 400 & 128 & 1024 & 64 & 128 \\
\hline \multicolumn{6}{|l|}{ Mutations $^{\mathrm{b}}$} \\
\hline \multicolumn{6}{|l|}{ PC2-TM7 control } \\
\hline R558C & 400 & 128 & 1024 & 64 & 128 \\
\hline E839C & 400 & 128 & 1024 & 64 & 128 \\
\hline R558C_E839C & 400 & 128 & 1024 & 64 & 128 \\
\hline \multicolumn{6}{|l|}{ PC2-TM7 } \\
\hline S562C & 200 & $64-128$ & 512 & $32-64$ & 64 \\
\hline T837C & 400 & 128 & 1024 & 64 & $64-128$ \\
\hline S562C_T837C & 50 & 32 & 256 & $16-32$ & 32 \\
\hline \multicolumn{6}{|l|}{$P N 1-P N 2$} \\
\hline S132C & 200 & 128 & 1024 & 64 & $64-128$ \\
\hline A294C & 400 & 128 & 1024 & $32-64$ & 128 \\
\hline S132C_A294C & 50 & $16-32$ & 256 & 32 & $8-16$ \\
\hline \multicolumn{6}{|l|}{ PN2-TM1 } \\
\hline V32C & 400 & 64 & 1024 & 64 & 128 \\
\hline N298C & 50 & 16 & 256 & 16 & 16 \\
\hline V32C_N298C & 25 & 16 & 128 & 16 & 16 \\
\hline A299C & 400 & 128 & 1024 & 64 & 128 \\
\hline V32C_A299C & 400 & 64 & 1024 & 64 & 64 \\
\hline \multicolumn{6}{|l|}{$100 p^{c}-D C$} \\
\hline s233C & 400 & 128 & 1024 & 64 & 128 \\
\hline Q726C & 400 & 128 & 1024 & $32-64$ & 128 \\
\hline S233C_Q726C & $200-400$ & 128 & 1024 & $32-64$ & 128 \\
\hline $1235 \mathrm{C}$ & 400 & 128 & 1024 & 64 & 128 \\
\hline K728C & 400 & 128 & 1024 & $32-64$ & 128 \\
\hline 1235C_K728C & 400 & $64-128$ & 1024 & 64 & 128 \\
\hline V225C & 400 & $64-128$ & 1024 & 64 & 128 \\
\hline A777C & 400 & $64-128$ & 1024 & 64 & 128 \\
\hline V225C_A777C & 400 & 64 & 1024 & 64 & 128 \\
\hline \multicolumn{6}{|l|}{$1000-P C 1$} \\
\hline Q229C & 400 & 128 & 1024 & 64 & 128 \\
\hline T583C & $100-200$ & $32-64$ & 51.2 & 32 & 64 \\
\hline Q229C_T583C & 50 & $8-16$ & 256 & 16 & 16 \\
\hline R586C & 400 & $64-128$ & 1024 & 32 & $64-128$ \\
\hline Q229C_R586C & $100-200$ & 32 & 512 & $16-32$ & $32-64$ \\
\hline
\end{tabular}

${ }^{\mathrm{a}}$ E. coli BW25113 $\mathrm{acrB}$ harboring pET24a or PET24acrB_cl (encoding AcrB devoid of cysteines) were used as a negative or positive control, respectively. "The numbers in boldface, italic type are the minimal inhibitory concentration (MIC) values that were reduced by at least four-fold compared to the single-cysteine mutant showing the lowest MIC value(s). Cysteine residues are located on the subdomains or regions indicated in italics. Intermonomer connecting loop. Each assay was repeated at least three times.

could account for approximately 50\% cross-linked AcrB trimers and in this way also account for the observed inhibition of activity.

Another strong indication for the presence of the $\mathrm{O}$ conformation has been shown recently by the specific binding of designed ankyrin repeat proteins (DARPins) to the $\mathrm{L}$ and $\mathrm{T}$ monomers, but not to the 

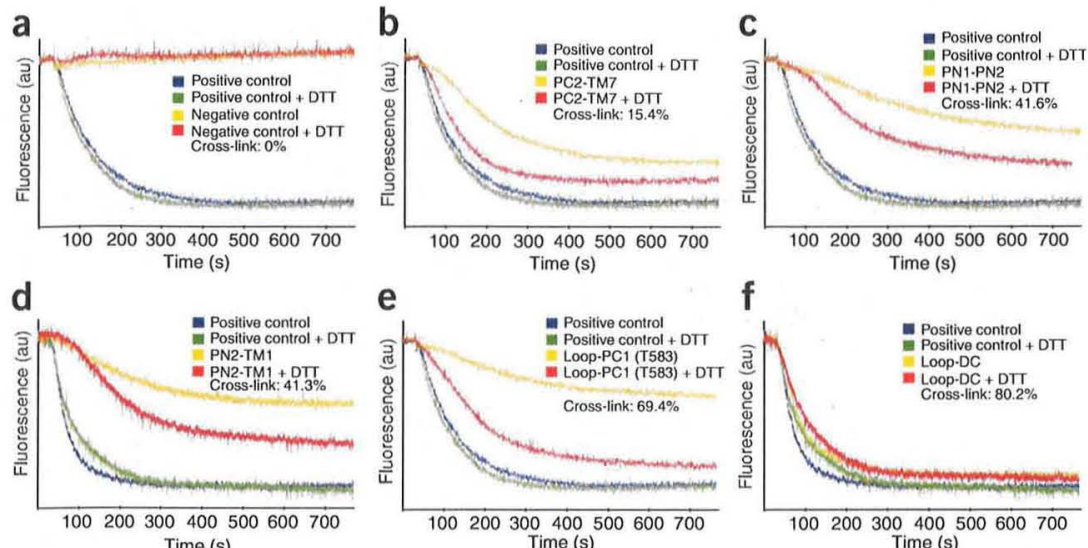

Figure 3 Effect of DTT on N-phenylnaphthylamine (NPN) efflux by $E$. coli BW25113 1 acrB producing AcrB_cl mutants with disulfide links between the indicated subdomains. (a) AcrB_cl was used as a positive control, and the nonfunctional D407N_D408N mutant as a negative control. (b-e) Mutants with cross-links between the PC2 subdomain and TM7 (S562C_T837C; b), the PN1 and PN2 subdomains (S132C_A294C; c), the PN2 subdomain and TM1 (V32C_N298C; d) and the loop and PC1 subdomain (Q229C_T583C; e) have reduced NPN efflux activity, and addition of DTT restores in part the activity by reducing the disulfide cross-links. (f) The NPN efflux activity of the loop-DC subdomain (V225C_A777C) cross-linked mutant was almost unaffected. Minimal inhibitory concentration (MIC) determinations confirm these results (Table 2 and Supplementary Fig. 2). au, arbitrary units.

O monomer, owing to structural constraints ${ }^{19}$. Analytical ultracentrifugation confirmed the binding of two DARPins to trimeric AcrB. Therefore, trimeric AcrB in detergent solution seems to be present predominantly in an asymmetric conformation, because the symmetric conformation would be expected to bind three DARPin molecules per trimeric AcrB.

The formation of disulfide bridges between AcrB monomers using cysteine-substituted Val105 and Gln112 was reported in a study in which amino acid residues Asp99 to Pro119 of the pore helix were systematically exchanged for cysteines ${ }^{25}$. The cross-linking results were interpreted according to the symmetric structure, but could not explain the explicit preference for the V105C or Q112C intermolecular crosslinking or the lack of cross-link in the $\mathrm{N} 109 \mathrm{C}$ mutant as, based on the symmetric AcrB structure, this residue would be within the suitable cross-linking range of below $6.4 \AA$. Interestingly, according to the asymmetric structure, only Val105 and Gln 112 have an appropriate distance for cross-linking, but not Asn109 (Supplementary Table 1 online). In accordance, the cross-linked V115C and Q112C mutants conferred a global reduction of MIC for all substrates tested ${ }^{25}$. These results can be taken as another line of evidence that AcrB trimer exists, probably exclusively, in an asymmetric conformation in the membrane.

The observed amount of cross-linking for the PN2-TM1 and PN1PN2 mutants (41-42\%) suggests that more than one monomer per trimer is cross-linked. Assuming that no cross-linking occurs at distances above the theoretical value of $6.4 \AA$ between the $S \gamma$ atoms, it must be concluded that more than one monomer in the trimer can adopt the $\mathrm{L}$ or $\mathrm{T}$ conformation, at least in the PN1-PN2 or PN2-TM1 mutant, respectively. This result implies that the AcrB trimer has the flexibility to comprise more than one monomer in the same conformational state. There is a clear agreement between the MIC values and NPN efflux activity for the PN1-PN2, PC2-TM7, loop-DC and loop-PC1 mutants (Supplementary Fig. 2). For the PN2-TM1 (V32C_N298C) mutant, the recovery of the NPN efflux activity upon treatment with DTT clearly showed the inhibition of V32C_N298C cross-link formation in addition to the inhibitory effect of the N298C mutation, whereas the reduction of MIC values due to disulfide bond formation was observed only by a two-fold reduction in MIC for TPP and berberine. Cross-linking the PC1 subdomain with the loop leads to a large reduction of MIC values for all substrates tested (Table 2). This reduction was slightly more for the Q229C_T583C mutant than for the Q229C_R586C mutant, which might correlate with the amount of cross-linking observed (69\% and $46 \%$, respectively; Table 1 ). The PC1 subdomain contains residues (Phe610, Phe615, Phe617, Phe628, Val612 and Ile626) constituting part of the hydrophobic binding pocket that is present exclusively in the T monomer (Supplementary Fig. 4 online). Restriction of the subtle PC1 subdomain movement in the loop-PC1 mutant results in a considerable loss of activity, which might reflect the importance of this subdomain movement for binding and transporting drugs. In summary, the data presented here provide evidence for an asymmetric conformation of AcrB in vivo and support the implied functional rotation of the AcrB trimer as constituting the monomer conformational cycling mechanism required for drug transport.

\section{METHODS}

Bacterial strains, plasmids and growth conditions. E. coli $\mathrm{DH} 5 \alpha^{26}$ and $E$. coli Mach1-T1 (Invitrogen) were routinely used as hosts for cloning procedures. E. coli $\mathrm{C} 43(\mathrm{DE} 3)^{27}$ harboring $\mathrm{pET} 24 \mathrm{acrB} \mathrm{His}^{17}$ was used for $\mathrm{AcrB}$ overproduction. $\mathrm{LB}$ medium and $\mathrm{LB}$ agar ${ }^{28}$ were used for routine bacterial growth at $37^{\circ} \mathrm{C}$. Kanamycin (Applichem) was used at $50 \mu \mathrm{g} \mathrm{ml}^{-1}\left(\mathrm{Kan}^{50}\right)$.

Construction of acrB knockout. The acrB gene on the chromosome of $E$. coli BW25113 was deleted as described ${ }^{29}$. Drug resistance of $E$. coli BW25113 $\triangle a c r B$ could be fully restored by constitutive 'leaky' expression of acrB from the pET24acrB $\mathrm{His}_{\text {is }}$ plasmid.

Site-directed mutagenesis. A derivative of pET24acrB ${ }_{\mathrm{His}}$, pET24acrB_cl, encodes a cysteine-free variant of AcrB (C493A_C887A), which we designated AcrB_cl. It was used as a template for single- and double-cysteine substitutions. Site-directed mutagenesis was done using the Quikchange protocol (Stratagene). All cysteine substitutions were verified by sequencing and, for clones comprising the double-cysteine mutations, the entire $a c r B$ gene was sequenced.

Drug susceptibility assays. Determination of the minimal inhibitory concentration (MIC) was done as follows. Aliquots $(1.5 \mu \mathrm{l})$ of precultures of E. coli BW25113 $\triangle a c r B$ carrying pET24acrB cl with and without cysteine substitutions grown in $\mathrm{LB} \mathrm{Kan}^{50}$ (4 ml, final $\mathrm{OD}_{600}$ between 0.5 and 1) were used to inoculate LB $\operatorname{Kan}^{50}(150 \mu \mathrm{l})$ with two-fold serial dilutions of the indicated drug in wells of a 96-well microtiter plate. After incubation $\left(37^{\circ} \mathrm{C}\right.$ and $160 \mathrm{rev} \mathrm{min}^{-1}$ ) of $22-24 \mathrm{~h}$, the $\mathrm{OD}_{600}$ was determined. Control growth without added drugs lead to a maximum $\mathrm{OD}_{600}$ of 1.8-2.0, and drug concentration of samples with an $\mathrm{OD}_{600}$ of less than 0.58 (turbidity visual detection limit) was considered as minimal inhibitory concentration (MIC). Each assay was repeated at least three times.

$N$-Phenylnaphthylamine (NPN) efflux assay. Cultures of E. coli BW25113 $\triangle a c r B$ harboring pET24acrB_cl with and without cysteine mutations

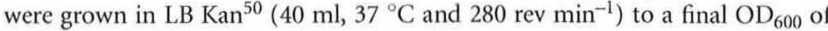
between 0.85 and 1.1, harvested, and resuspended in $50 \mathrm{mM}$ potassium phosphate buffer $\left(\mathrm{KP}_{\mathrm{i}}\right)$, pH 7, $1 \mathrm{mM} \mathrm{MgSO}_{4}(4 \mathrm{ml})$. Cells were de-energized 
in the presence of carbonyl cyanide $m$-chlorophenylhydrazone (CCCP; $200 \mu \mathrm{M}$ ) for $10 \mathrm{~min}$ on ice. CCCP was removed by washing the cells three times with $50 \mathrm{mM} \mathrm{KP}_{\mathrm{i}}, \mathrm{pH} 7,1 \mathrm{mM} \mathrm{MgSO}_{4}(4 \mathrm{ml})$. Cell density was adjusted to an $\mathrm{OD}_{600}$ of 0.2 , and NPN $(10 \mu \mathrm{M})$ and, if required, DL-DTT $(10 \mathrm{mM})$ were added. Incorporation of NPN into the inner membrane was followed by measuring fluorescence (excitation: $355 \mathrm{~nm}$, emission: $420 \mathrm{~nm}$ ) until saturation was reached $(6 \mathrm{~min})$. Cells were re-energized by addition of glucose $(0.2 \%(\mathrm{w} / \mathrm{v})$ final concentration) and the fluorescence signal was followed for another $12 \mathrm{~min}$

SDS-PAGE. Double-cysteine mutants of AcrB_cl were overproduced and purified as described ${ }^{9,17}$. Before solubilization, membranes were incubated with $\mathrm{N}$-ethylmaleimide (NEM, Sigma; $5 \mathrm{mM}$ final concentration) for $30 \mathrm{~min}$ at room temperature. Purified protein $(2 \mu \mathrm{g})$ was subjected to SDS-PAGE ${ }^{30}$ in the presence or absence of $\beta$-mercaptoethanol and stained with colloidal coomassie ${ }^{31}$

Western blot analysis. Cultures of E. coli BW25113 $\triangle a c r B$ carrying pET24acrB cl with and without additional mutations were grown in LB $\operatorname{Kan}^{50}(4 \mathrm{ml})$ to an $\mathrm{OD}_{600}$ of $0.6-1.0$ before harvest $(0.8 \mathrm{ml})$. The pellet was resuspended in SDS-PAGE loading buffer $\left(0.05 \mathrm{ml} \times \mathrm{OD}_{600}\right.$ of the harvested culture) including $2 \%(\mathrm{v} / \mathrm{v}) \beta$-mercaptoethanol and boiled for $5 \mathrm{~min}$. Under nonreducing conditions, NEM (5 $\mathrm{mM}$ final concentration) replaced $\beta$-mercaptoethanol in the loading buffer and samples were incubated for $30 \mathrm{~min}$ at room temperature before boiling. Each sample $(20 \mu \mathrm{l})$ was subjected to SDS-PAGE ${ }^{30}$ and blotted onto nitrocellulose membrane ${ }^{32}$. AcrB_cl and its mutant derivatives were immunodetected with anti-AcrB rabbit antibodies (Neosystem) at a 1:10 ${ }^{4}$ dilution. Bound immunoglobulins were probed with mouse anti-rabbit antibody coupled to horseradish peroxidase and visualized using the enhanced chemiluminescence ECL system (Pierce).

Quantification of disulfide bridges by mass spectrometry. An overview of the workflow is presented in Supplementary Figure 3. Membranes of E. coli C43 (DE3) containing overproduced double-cysteine mutants of AcrB $\mathrm{cl}$ were solubilized in the presence of NEM, and the detergent extract was applied on a nickel-nitrilotriacetic acid $\left(\mathrm{Ni}^{2+}{ }_{-} \mathrm{NTA}\right)$ gravity flow column (Qiagen). The free thiol groups were alkylated on the column using NEM $(10 \mathrm{mM})$ in the presence of guanidine hydrochloride $(2 \mathrm{M})$. The disulfide linkages were reduced using DTT $(10 \mathrm{mM})$ and the newly generated free thiol groups were modified, after removal of DTT, with N-methylmaleimide (NMM). Alternatively, the protoco was carried out starting with the NMM modification, followed by reduction of the disulfide bonds and NEM modification. The NEM- and NMM-treated mutant proteins were subsequently purified, and digested using cyanogen bromide $(\mathrm{CNBr})$ and trypsin before analysis using MALDI-TOF ${ }^{33}$. The signal-to-noise ratio of the peaks originating from peptides labeled with NEM and NMM were used for the quantification of the disulfide bonds.

Other methods. More detailed descriptions of the construction of the acrB knockout, the NPN efflux assay and the quantification of disulfide bridges by MS is provided in the Supplementary Methods online.

\section{ACKNOWLEDGMENTS}

MALDI-TOF measurements were performed at the Functional Genomics Center Zurich (FGCZ). We thank P. Gehrig from the FGCZ for assistance with the MALDI-TOF instrument. This work was supported by grants of the TR-SFB Zürich-Konstanz (to K.D.), the ETH Zürich, EMDO Stiftung and the FK of the University of Zürich (to K.M.P.).

\section{AUTHOR CONTRIBUTIONS}

M.A.S., C.v.B. and K.M.P. designed the experiments; M.A.S., C.v.B, T.E. and L.B. performed the experiments; M.A.S., F.V., K.D. and K.M.P. analyzed the data; M.A.S., K.D. and K.M.P. wrote the article.

1. Poole, K. Efflux-mediated multiresistance in Gram-negative bacteria. Clin. Microbiol. Infect. 10, 12-26 (2004).
2. Zgurskaya, H.I. \& Nikaido, H. Multidrug resistance mechanisms: drug efflux across two membranes. Mol. Microbiol. 37, 219-225 (2000).

3. Murakami, S. \& Yamaguchi, A. Multidrug-exporting secondary transporters. Curr. Opin. Struct. Biol. 13, 443-452 (2003).

4. Eswaran, J., Koronakis, E., Higgins, M.K., Hughes, C. \& Koronakis, V. Three's company component structures bring a closer view of tripartite drug efflux pumps. Curr. Opin Struct. Biol. 14, 741-747 (2004)

5. Koronakis, V., Sharff, A., Koronakis, E., Luisi, B. \& Hughes, C. Crystal structure of the bacterial membrane protein TolC central to multidrug efflux and protein export. Nature 405, 914-919 (2000)

6. Murakami, S., Nakashima, R., Yamashita, E. \& Yamaguchi, A. Crystal structure of bacterial multidrug efflux transporter AcrB. Nature 419, 587-593 (2002).

7. Pos, K.M., Schiefner, A., Seeger, M.A. \& Diederichs, K. Crystallographic analysis of AcrB. FEBS Lett. 564, 333-339 (2004).

8. Murakami, S., Nakashima, R., Yamashita, E., Matsumoto, T. \& Yamaguchi, A. Crystal structures of a multidrug transporter reveal a functionally rotating mechanism. Nature $443,173-179$ (2006).

9. Seeger, M.A. et al. Structural asymmetry of AcrB trimer suggests a peristaltic pump mechanism. Science 313, 1295-1298 (2006)

10. Mikolosko, J., Bobyk, K., Zgurskaya, H.I. \& Ghosh, P. Conformational flexibility in the multidrug efflux system protein AcrA. Structure 14, 577-587 (2006).

11. Andersen, C. et al. Transition to the open state of the TolC periplasmic tunnel entrance. Proc. Natl. Acad. Sci. USA 99, 11103-11108 (2002).

12. Tikhonova, E.B. \& Zgurskaya, H.I. AcrA, AcrB, and TolC of Escherichia coli form a stable intermembrane multidrug efflux Complex. J. Biol. Chem. 279, 32116-32124 (2004)

13. Touze, T. et al. Interactions underlying assembly of the Escherichia coli AcrAB-TolC multidrug efflux system. Mol. Microbiol. 53, 697-706 (2004).

14. Lobedanz, S. et al. A periplasmic coiled-coil interface underlying TolC recruitment and the assembly of bacterial drug efflux pumps. Proc. Natl. Acad. Sci. USA 104, 4612-4617 (2007)

15. Tikhonova, E.B., Wang, Q. \& Zgurskaya, H.I. Chimeric analysis of the multicomponen multidrug efflux transporters from Gram-negative bacteria. J. Bacteriol. 184 6499-6507 (2002)

16. Eda, S., Maseda, H. \& Nakae, T. An elegant means of self-protection in Gram-negative bacteria by recognizing and extruding xenobiotics from the periplasmic space. J. Biol. Chem. 278, 2085-2088 (2003).

17. Pos, K.M. \& Diederichs, K. Purification, crystallization and preliminary diffraction studies of AcrB, an inner-membrane multi-drug efflux protein. Acta Crystallogr. D Biol. Crystallogr. 58, 1865-1867 (2002).

18. Yu, E.W., McDermott, G., Zgurskaya, H.I., Nikaido, H. \& Koshland, D.E., Jr. Structura basis of multiple drug-binding capacity of the AcrB multidrug efflux pump. Science 300, 976-980 (2003).

19. Sennhauser, G., Amstutz, P., Briand, C., Storchenegger, O. \& Grutter, M.G. Drug export pathway of multidrug exporter AcrB revealed by DARPin inhibitors. PLoS Biol. 5, Acta Crystallogr. D Biol. Crystallogr. 5, e7 (2007)

20. Abrahams, J.P., Leslie, A.G., Lutter, R. \& Walker, J.E. Structure at $2.8 \mathrm{~A}$ resolution of F1-ATPase from bovine heart mitochondria. Nature 370, 621-628 (1994).

21. Nikaido, $\mathrm{H}$. Multidrug efflux pumps of Gram-negative bacteria. J. Bacteriol. 178 5853-5859 (1996).

22. Lomovskaya, 0 . et al. Identification and characterization of inhibitors of multidrug resistance efflux pumps in Pseudomonas aeruginosa: novel agents for combination therapy. Antimicrob. Agents Chemother. 45, 105-116 (2001).

23. Colonna-Cesari, F. \& Sander, C. Excluded volume approximation to protein-solvent interaction. The solvent contact model. Biophys. J. 57, 1103-1107 (1990).

24. Dombkowski, A.A. \& Crippen, G.M. Disulfide recognition in an optimized threading potential. Protein Eng. 13, 679-689 (2000)

25. Murakami, S., Tamura, N., Saito, A., Hirata, T. \& Yamaguchi, A. Extramembrane central pore of multidrug exporter AcrB in Escherichia coli plays an important role in drug transport. J. Biol. Chem. 279, 3743-3748 (2004).

26. Woodcock, D.M. et al. Quantitative evaluation of Escherichia coli host strains for tolerance to cytosine methylation in plasmid and phage recombinants. Nucleic Acids Res. 17, 3469-3478 (1989).

27. Miroux, B. \& Walker, J.E. Over-production of proteins in Escherichia coli: mutant hosts that allow synthesis of some membrane proteins and globular proteins at high levels. $J$. Mol. Biol. 260, 289-298 (1996).

28. Sambrook, J., Fritsch, E.F. \& Maniatis, T. Molecular Cloning: A Laborator Manual (Cold Spring Harbor Laboratory Press, Cold Spring Harbor, New York, USA, 1989).

29. Datsenko, K.A. \& Wanner, B.L. One-step inactivation of chromosomal genes in Escherichia coli K-12 using PCR products. Proc. Natl. Acad. Sci. USA 97 $6640-6645$ (2000)

30. Schagger, H. \& von Jagow, G. Tricine-sodium dodecyl sulfate-polyacrylamide ge electrophoresis for the separation of proteins in the range from 1 to $100 \mathrm{kDa}$. Anal. Biochem. 166, 368-379 (1987).

31. Molloy, P.L. Electrophoretic mobility shift assays. Methods Mol. Biol. 130, 235-246 (2000).

32. Kyhse-Andersen, J. Electroblotting of multiple gels: a simple apparatus without buffer tank for rapid transfer of proteins from polyacrylamide to nitrocellulose. J. Biochem Biophys. Methods 10, 203-209 (1984).

33. Kraft, P. Mills, J. \& Dratz, E. Mass spectrometric analysis of cyanogen bromide fragments of integral membrane proteins at the picomole level: application to rhodopsin. Anal. Biochem. 292, 76-86 (2001). 